Service social

\title{
Les jeunes, leur sexualité et la prévention du sida : une problématique et un défi social
}

\section{Marie Drolet}

Volume 38, numéro 1, 1989

Aspects psychosociaux du Sida

URI : https://id.erudit.org/iderudit/706424ar

DOI : https://doi.org/10.7202/706424ar

Aller au sommaire du numéro

Éditeur(s)

École de service social de l'Université Laval

ISSN

1708-1734 (numérique)

Découvrir la revue

Citer cet article

Drolet, M. (1989). Les jeunes, leur sexualité et la prévention du sida : une problématique et un défi social. Service social, 38(1), 61-77.

https://doi.org/10.7202/706424ar
Résumé de l'article

Cette recension des écrits examine les connaissances, attitudes et comportements des jeunes vis-à-vis du sida et considère les développements actuels en matière d'évaluation des programmes de prévention auprès de cette même population. 
Drolet, Marie, étudiante au doctorat, École de service social de l'Université Laval.

\section{Les jeunes, leur sexualité et la prévention du sida : une problématique et un défi social}

\section{Marie Drolet}

Selon une étude divulguée en juin 1989 par le Center for Disease Control d'Atlanta, les adolescents et adolescentes de 13 à 19 ans représentent aux États-Unis $0.4 \%$ des 87,188 cas de sida (morts ou vivants) officiellement recensés depuis 1981. De leur côté, les jeunes adultes de 20 à 24 ans désignent $4.4 \%$ et ceux de 25 à 29 ans totalisent par contre $16.2 \%$ (Lévesque, 1989). Certes, le temps d'incubation du virus d'immunodéficience humaine (VIH) se prolongeant parfois au-delà de sept ans, les pourcentages concernant les jeunes adultes actuellement atteints peuvent notamment être des conséquences de l'activité sexuelle durant l'adolescence.

En outre, Sorenson caractérise cette activité sexuelle chez les jeunes par cinq spécificités (cité dans Frappier, 1980; Wilkins, 1985 ; Desrosiers, 1986 ; Hein, 1987 ; Sunenblick, 1988). Elle est progressive, non planifiée, sporadique sauf lors d'une relation intense prolongée, mais surtout sélective et successive selon le modèle de la monogamie en série ; finalement, il y a poursuite de cette activité sexuelle après la première relation coïtale. Par ailleurs, l'usage de la drogue et de l'alcool aurait un impact en faveur d'une sexualité non planifiée et non protégée (King et al., 1988).

Parmi ces éléments distinctifs, la succession de partenaires justifie de cibler les jeunes comme une population à privilégier pour des campagnes préventives sur le sida. En effet, puisque cette maladie n'est plus restreinte à des groupes préalablement désignés comme "à risques " (les homosexuels et les consommateurs de drogues intraveineuses), la prévention vise maintenant à défaire cette image des groupes d'origine, pour davantage informer sur les comportements à risques et sensibiliser les personnes les plus concernées, soit celles présentant de tels agirs.

S.S., vol. 38, no 1, 1989. 
De plus, à partir de considérations similaires, le Centre fédéral sur le sida a initié en 1987 une Étude sur les jeunes Canadiens face au SIDA afin de cerner leurs connaissances, attitudes et comportements sexuels vis-à-vis du VIH et des autres maladies transmissibles sexuellement (MTS). Les résultats de cette recherche (King et al., 1988) publicisés en février 1989 (Bellefeuille, 1989), et particulièrement le volet décrivant la situation au Québec (Beazley et al., 1988), deviendront l'assise de cette mise au point en matière de prévention du sida en regard de la sexualité adolescente.

\section{Les connaissances, attitudes et comportements sexuels des jeunes en regard du sida}

Tout d'abord, il est convenu de fixer l'âge moyen du premier rapport coïtal à 15-16 ans (Frappier, 1980 ; Tessier, 1985; Wilkins, 1985; Desrosiers, 1986). Plus précisément au Québec, la recherche de Filion (1986) réalisée auprès de 1,072 étudiants et étudiantes de la Commission des écoles catholiques de Montréal des secondaires III, IV et V, montre que $40.1 \%$ des adolescents sont actifs sexuellement. Les données recueillies par Beazley et al. (1988) clarifient ce taux global. En effet, $47 \%$ des répondants de secondaire $V$ ont déjà eu une relation sexuelle et $61 \%$ d'entre eux avec au moins deux partenaires ; pourtant en secondaire III, ils ne sont que $28 \%$ à être sexuellement actifs.

Quant aux notions spécifiques à la problématique du sida, le tableau 1 illustre onze études récentes sur les connaissances, attitudes et comportements sexuels des jeunes vis-à-vis de cette nouvelle réalité.

Au plan des connaissances, les jeunes Québécois présentent des résultats comparables à ceux de l'ensemble des jeunes Canadiens (Beazley et al., 1988). De fait, les pourcentages globaux des questions auxquelles ont répondu correctement les étudiants du Québec des secondaires I, III et $V$, peuvent être mis en parallèle avec ceux des étudiants canadiens de $7^{\mathrm{e}}, 9^{\mathrm{e}}$ et $11^{\mathrm{e}}$ années : les premiers réussissent à des taux de $70 \%, 77 \%$ et $67 \%$ d'exactitude de connaissances, les seconds obtiennent $68 \%, 78 \%$ et $67 \%$. Un portrait similaire se dégage quant à la question portant sur la transmission du VIH lors de relations hétérosexuelles ; les Québécois atteignent alors $91 \%, 89 \%$ et $94 \%$ de réponses exactes (Canada : $90 \%, 91 \%$ et $93 \%$ ).

Si ces résultats sont interprétés à l'aide du tableau 1, les Québécois et les Canadiens ont un niveau de connaissance sur la transmission sexuelle du VIH analogue à celui des jeunes américains. En effet, DiClemente et al. (1986) avancent que $92 \%$ des répondants savent que le sida se contracte lors de relations sexuelles, $95 \%$ d'après Gottlieb et 
al. (1988). Pour Strunin et Hingson (1987) de même que Winslow (1988), un taux similaire concerne les relations hétérosexuelles; selon Sunenblick (1988), 96\% sont informés que le VIH se transmet par voie anale, 93\% connaissent la transmission vaginale.

Quand on analyse l'ensemble des connaissances sur le sida et non plus seulement sur les modes de transmission sexuelle, on constate que les jeunes Québécois et Canadiens ont un niveau de sensibilisation modéré (de 67 à $78 \%$ de réponses adéquates). Il est pourtant supérieur à celui des étudiants de l'Ohio (Price et al., 1985) et du Michigan (Goodwin et Roscoe, 1988). Il est à noter que les plus faibles résultats touchent l'incurabilité du sida (par exemple : $74 \%$ des Québécois de secondaire $V$ ont la bonne réponse) et l'absence de symptômes pendant une période de sept ans ou plus (seulement $62 \%$ de ce même groupe répondent adéquatement). Ces notions sont pourtant centrales pour saisir le contexte de risque inhérent au VIH.

Même si tout cet état de la question vise une discussion autour de la sexualité hétérosexuelle des adolescents et adolescentes, il importe de mentionner, à titre indicatif, le niveau de connaissances sur le mode de transmission par échange de seringues, vu l'usage de la drogue chez les jeunes. Au Québec, $92 \%$ des étudiants de secondaire $V$ ont une réponse exacte; au Canada, les taux sont de $93 \%$ en $9^{\mathrm{e}}$ année et de $96 \%$ en $11^{\mathrm{e}}$ année. Ces pourcentages sont comparables au $98 \%$ recueilli par Gottlieb et al. (1988). Par contre, les Québécois de secondaire III (84\%) et les Canadiens de $7^{\mathrm{e}}$ année ( $83 \%$ ) ont un taux similaire au $81 \%$ soutenu par DiClemente et al. (1986) et Goodwin et Roscoe (1988). Les étudiants de secondaire I présentent toutefois un taux inférieur à tous, soit seulement $71 \%$ de bonnes réponses.

Sachant que le virus du VIH se transmet lors de relations hétérosexuelles, les jeunes adopteront-ils des comportements de protection (Safer Sex), comme l'utilisation régulière du condom ? King et al. (1988) ont posé cette question uniquement aux décrocheurs et aux étudiants postsecondaires. Moins de $20 \%$ d'entre eux utilisent toujours le condom, plus de $25 \%$ ne l'utilisent jamais. Ces pourcentages se comparent à ceux soulevés par Strunin et Hingson (1987) et par Sunenblick (1988). En effet, selon les premiers, $70 \%$ des répondants sont sexuellement actifs et de ceux-ci, $20 \%$ ont adopté des comportements effectivement protecteurs, tel le préservatif ; d'après Sunenblick, $31 \%$ des filles et $27 \%$ des garçons ont choisi le condom comme méthode contraceptive. Par ailleurs, DiClemente et al. (1986) affirment que seulement $60 \%$ savent que le condom peut les protéger. De plus, $27 \%$ des étudiants du Texas ne pratiquent jamais de "Safer Sex " et $33 \%$ affirment que leur partenaire le refuse (Gottlieb et al., 1988). D'un autre côté, $51.6 \%$ des adultes montréalais qui ont un(e) partenaire occasionnel(le) et $75 \%$ vivant leur 


\section{TABLEAU 1}

Description des connaissances, attitudes et comportements sexuels des jeunes en regard du SIDA

\begin{tabular}{|c|c|c|c|c|c|c|c|c|}
\hline $\begin{array}{c}\text { Devis et } \\
\text { Recherches }\end{array}$ & $\begin{array}{l}\text { Objet } \\
\text { d'étude }\end{array}$ & \begin{tabular}{|l|} 
Date de \\
parution
\end{tabular} & $\begin{array}{c}\text { Date de } \\
\text { cueillette }\end{array}$ & \begin{tabular}{|c|} 
Lieu de \\
réalisation
\end{tabular} & $\begin{array}{l}\text { Groupe- } \\
\text { cible }\end{array}$ & $\begin{array}{l}\text { Échan- } \\
\text { tillon }\end{array}$ & $\begin{array}{l}\text { Cueillette } \\
\text { de données }\end{array}$ & Résultats \\
\hline Price et al. & $\begin{array}{l}\text { Mesurer les percep- } \\
\text { tions et les connais- } \\
\text { sances sur le SIDA }\end{array}$ & 1985 & 1984 & Ohio & $\begin{array}{l}\text { Étudiants du } \\
\text { secondaire } \\
\text { (16-19 ans) }\end{array}$ & 250 & $\begin{array}{l}\text { - Questionnaire en } \\
\text { classe }\end{array}$ & $\begin{array}{l}\text { - De } 50 \% \text { à } 75 \% \text { des répondants ont certaines connaissances } \\
\text { sur le SIDA, mais moins sur les modes de transmission; les } \\
\text { mieux informés ont } 47 \% \text { de bonnes réponses. } \\
\text { - } 73 \% \text { ne sont pas personnellement préoccupés d'avoir le } \\
\text { SIDA éventuellement. }\end{array}$ \\
\hline $\begin{array}{l}\text { Strunin et } \\
\text { Hingson }\end{array}$ & $\begin{array}{l}\text { Mesurer les connais- } \\
\text { sances, les attitudes, les } \\
\text { croyances et les com- } \\
\text { portements sur le SIDA }\end{array}$ & 1987 & 1986 & $\begin{array}{l}\text { Massa- } \\
\text { chusetts }\end{array}$ & $\begin{array}{l}\text { Adolescents } \\
\text { (16-19 ans) }\end{array}$ & $\begin{array}{c}829 \\
\text { (partici- } \\
\text { pation de } \\
86 \% \text { ) }\end{array}$ & $\begin{array}{l}\text { - Sondage par } \\
\text { téléphone }\end{array}$ & $\begin{array}{l}\text { - 92\% savent que le SIDA se transmet lors de relations } \\
\text { hétérosexuelles (aucune différence chez les répondants } \\
\text { actifs ou non actifs sexuellement). } \\
\text { - } 70 \% \text { sont sexuellement actifs; } 15 \% \text { ont déjà changé leurs } \\
\text { agirs en raison du SIDA, mais seulement } 20 \% \text { avec des } \\
\text { comportements effectivement protecteurs. } \\
\text { - } 54 \% \text { ne sont pas préoccupés d'avoir le SIDA un jour. }\end{array}$ \\
\hline
\end{tabular}




\begin{tabular}{|c|c|c|c|c|c|c|c|c|}
\hline $\begin{array}{l}\text { Goodwin } \\
\text { et Roscoe }\end{array}$ & $\begin{array}{l}\text { Mesurer les connais- } \\
\text { sances et les attitudes } \\
\text { vis-à-vis du SIDA }\end{array}$ & 1988 & 1985 & Michigan & $\begin{array}{l}\text { Étudiants du } \\
\text { collège en } \\
\text { majorité de } \\
\text { classe } \\
\text { moyenne et } \\
\text { de race } \\
\text { blanche }\end{array}$ & 495 & $\begin{array}{l}\text { - Questionnaire de } \\
49 \text { items: } \\
\text { connaissances sur } \\
\text { le SIDA, peur de } \\
\text { le contracter et } \\
\text { attitudes vis-à-vis } \\
\text { de } \\
\text { l'homosexualité }\end{array}$ & $\begin{array}{l}\text { - Sur } 21 \text { des } 32 \text { items de connaissances, } 2 / 3 \text { des répondants } \\
\text { ont la bonne réponse. } \\
\text { - } 45 \% \text { sont très peu acceptants vis-à-vis de l'homosexualité; } \\
\text { les plus acceptants ont aussi moins peur de contracter le } \\
\text { SIDA. } \\
\text { - } 68 \% \text { disent se sentir relativement concernés. }\end{array}$ \\
\hline Winslow & $\begin{array}{l}\text { Construire un ques- } \\
\text { tionnaire puis mesurer } \\
\text { les connaissances sur } \\
\text { les modes de transmis- } \\
\text { sion du SIDA }\end{array}$ & 1988 & 1986 & San Diego & $\begin{array}{l}\text { Étudiants non } \\
\text { gradués } \\
\text { universitaires }\end{array}$ & 375 & $\begin{array}{l}\text { - Questions } \\
\text { ouvertes remplies } \\
\text { en classe } \\
\text { - Puis élaboration } \\
\text { d'un question- } \\
\text { naire fermé } \\
\text { administré par la } \\
\text { suite en classe }\end{array}$ & $\begin{array}{l}\text { - La majorité a une connaissance adéquate des modes de } \\
\text { transmission. } \\
\text { - Ce concept devrait être subdivisé en } 4 \text { dimensions : contacts } \\
\text { "secs » (toucher), contacts " humides " (utilisation d'un verre), } \\
\text { contacts de la salive (baisers), relations sexuelles. }\end{array}$ \\
\hline $\begin{array}{l}\text { Gottlieb } \\
\text { et al. }\end{array}$ & $\begin{array}{l}\text { Mesurer les connais- } \\
\text { sances, attitudes, } \\
\text { croyances et comporte- } \\
\text { ments en regard du } \\
\text { SIDA }\end{array}$ & 1988 & 1987 & Texas & $\begin{array}{l}\text { Étudiants de } \\
\text { quatre } \\
\text { collèges }\end{array}$ & $\begin{array}{c}670 \\
\text { (partici- } \\
\text { pation de } \\
34 \% \text { ) }\end{array}$ & $\begin{array}{l}\text { - Sondage par la } \\
\text { poste } \\
\text { - Échelle Likert de } \\
50 \text { items sur leurs } \\
\text { activités } \\
\text { sexuelles, le } \\
\text { "Safer Sex", les } \\
\text { modes d'informa- } \\
\text { tion sur le SIDA }\end{array}$ & $\begin{array}{l}\text { - } 95 \% \text { connaissent les modes de transmission du SIDA et } \\
\text { l'utilisation du condom. } \\
-27 \% \text { ne pratiquent jamais de "Safer Sex». } \\
-94 \% \text { ne se considèrent pas un groupeà hauts risques, ayant } \\
\text { des relations sexuelles avec des partenaires connus ( } 85 \%) \\
\text { et en relation stable }(53 \%) \text {. } \\
-70 \% \text { sont peu/pas intéressés à des ateliers de "Safer Sex». }\end{array}$ \\
\hline
\end{tabular}


TABLEAU 1

Description des connaissances, attitudes et comportements sexuels des jeunes en regard du SIDA (suite)

\begin{tabular}{|c|c|c|c|c|c|c|c|c|}
\hline $\begin{array}{c}\text { Devis et } \\
\text { Recherches }\end{array}$ & $\begin{array}{c}\text { Objet } \\
\text { d'étude }\end{array}$ & $\begin{array}{l}\text { Date de } \\
\text { parution }\end{array}$ & $\begin{array}{l}\text { Date de } \\
\text { cueillette }\end{array}$ & \begin{tabular}{|c|} 
Lieu de \\
réalisation
\end{tabular} & $\begin{array}{l}\text { Groupe- } \\
\text { cible }\end{array}$ & $\begin{array}{l}\text { Échan- } \\
\text { tillon }\end{array}$ & $\begin{array}{l}\text { Cueillette } \\
\text { de données }\end{array}$ & Résultats \\
\hline $\begin{array}{l}\text { Clift et } \\
\text { Stears }\end{array}$ & $\begin{array}{l}\text { Mesurer la modifica- } \\
\text { tion de croyances et } \\
\text { d'attitudes à l'égard du } \\
\text { SIDA après une cam- } \\
\text { pagne publicitaire } \\
\text { nationale et scolaire }\end{array}$ & 1988 & $\begin{array}{l}1986 \\
\text { et } \\
1987\end{array}$ & $\begin{array}{l}\text { Canterbury } \\
\text { Angleterre }\end{array}$ & $\begin{array}{l}\text { Étudiants de } \\
\text { collèges }\end{array}$ & 184 & $\begin{array}{l}\text { - Questionnaire } \\
\text { rempli en classe: } \\
56 \text { items divisés } \\
\text { en } 2 \text { dimensions } \\
\text { "Worry index", } \\
\text { "Moral index" }\end{array}$ & $\begin{array}{l}\text { - Durant cette période, il y a eu une augmentation des } \\
\text { connaissances et une baisse des peurs par rapport aux } \\
\text { modes de transmission, mais pas de changements dans les } \\
\text { agirs sexuels. }\end{array}$ \\
\hline Sunenblick & $\begin{array}{l}\text { Mesurer les connais- } \\
\text { sances sur le SIDA et les } \\
\text { comportements sexuels } \\
\text { à risques }\end{array}$ & 1988 & 1987 & Maine & $\begin{array}{l}\text { Étudiants du } \\
\text { collège et de } \\
\text { l'université } \\
\text { (de } 17 \text { à } 23 \text { ans } \\
\text { avec une } \\
\text { moyenne de } \\
18 \text { ans) }\end{array}$ & 90 & $\begin{array}{l}\text { - Questionnaire } \\
\text { rempli en classe } \\
\text { (47 items) et basé } \\
\text { sur celui de } \\
\text { DiClemente et al. } \\
\text { (1986) en ce qui } \\
\text { concerne les } \\
\text { connaissances }\end{array}$ & $\begin{array}{l}\text { - Ils ont un haut niveau de connaissances des modes de } \\
\text { transmission sexuelle anale ( } 96 \% \text { ) et vaginale ( } 93 \%) \text {. } \\
\text { - Même si } 80 \% \text { considèrent avoir assez d'informations pour } \\
\text { se protéger, seulement } 31 \% \text { des filles et } 27 \% \text { des garçons } \\
\text { utilisent le condom comme contraceptif. } \\
\text { - } 50 \% \text { ont peur de contracter le virus. }\end{array}$ \\
\hline $\begin{array}{l}\text { Beazley } \\
\text { et al. }\end{array}$ & $\begin{array}{l}\text { Volet québécois de } \\
\text { l'étude canadienne de } \\
\text { King et al. }\end{array}$ & 1988 & 1988 & Québec & $\begin{array}{l}\text { Étudiants des } \\
\text { secondaires I, } \\
\text { III, V (analyse } \\
\text { par province) }\end{array}$ & 2756 & $\begin{array}{l}\text { - Questionnaire en } \\
\text { classe }\end{array}$ & $\begin{array}{l}\text { - Les Québécois ont des résultats comparables aux autres } \\
\text { Canadiens, par rapport aux connaissances, à la fréquence } \\
\text { des relations sexuelles et aux attitudes à l'endroit des } \\
\text { personnes atteintes. } \\
\text { - Ils ont des attitudes plus positives à l'égard du condom et } \\
\text { de l'homosexualité. } \\
\text { - } 50 \% \text { ont peur de contracter le SIDA. }\end{array}$ \\
\hline
\end{tabular}




\begin{tabular}{|c|c|c|c|c|c|c|c|c|}
\hline King et al. & $\begin{array}{l}\text { Analyser le développe- } \\
\text { ment des connais- } \\
\text { sances, des attitudes et } \\
\text { des comportements vis- } \\
\text { à-vis du SIDA et des } \\
\text { autres MTS }\end{array}$ & 1988 & 1988 & Canada & $\begin{array}{l}\text { Étudiants de } \\
7 \mathrm{e}, 9 \mathrm{ge}, 11 \mathrm{e} \\
\text { années et } 1 \text { e } \\
\text { universitaire } \\
\text { ou collégiale, } \\
\text { en abandon } \\
\text { scolaire, et } \\
\text { des itinérants } \\
\text { (11 à } 21 \text { ans) }\end{array}$ & 38002 & $\begin{array}{l}\text { - Questionnaire en } \\
\text { classe, entrevues } \\
\text { en profondeur } \\
\text { avec itinérants }\end{array}$ & $\begin{array}{l}\text { - Pourcentages moyens de réponses exactes sur la connais- } \\
\text { sance du SIDA : } 7 \text { e année (secondairel) : } 68 \% \text {; } 9 \text { e (sec. III): } \\
78 \% ; 11 \text { ( } \text { (sec. IV) : } 69 \% \text {; décrocheurs : } 77 \% \text {; post secon- } \\
\text { daire: } 72 \% \text {. } \\
\text { - Moins de } 20 \% \text { utilisent le condom. } \\
\text { - } 50 \% \text { ont peur " d'attraper » le SIDA. }\end{array}$ \\
\hline Greatorex & $\begin{array}{l}\text { Décrire les comporte- } \\
\text { ments sexuels pendant } \\
\text { les } 3 \text { mois précédents }\end{array}$ & 1989 & 1988 & $\begin{array}{l}\text { Manchester } \\
\text { Angleterre }\end{array}$ & $\begin{array}{l}\text { Étudiants de } \\
\text { 1e année } \\
\text { universitaire }\end{array}$ & $\begin{array}{c}264 \\
\text { (partici- } \\
\text { pation de } \\
43 \% \text { ) }\end{array}$ & $\begin{array}{l}\text { - Questionnaire } \\
\text { par la poste com- } \\
\text { portant } 15 \text { items }\end{array}$ & $\begin{array}{l}\text { - La majorité des relations sexuelles occasionnelles se font } \\
\text { sans condom. Ce type de relations ne représente que } 25 \% \\
\text { des contacts indiqués. }\end{array}$ \\
\hline
\end{tabular}


sexualité avec un amant régulier n'utilisent jamais le condom (Dupras et al., 1989).

En outre, les Québécois de secondaire $V$ ont une opinion plus positive à l'égard du condom que les jeunes Canadiens de $11^{\mathrm{e}}$ année ; certes, les avis demeurent relativement mitigés. En effet, $34 \%$ des élèves du Québec seraient gênés d'avoir à acheter des préservatifs (Canada : $40 \%$ ). Par contre, $46 \%$ d'entre eux (Canada : $53 \%$ ) pensent que le fait d'en avoir un avec soi signifie qu'on veut avoir une relation sexuelle.

Afin de faciliter l'intégration de comportements protecteurs chez les jeunes, il importe qu'ils se sentent concernés par la problématique (Strunin, 1989) ; les pourcentages subséquents représentent donc une certaine entrave à l'adoption de gestes préventifs. En effet, seulement "environ la moitié des jeunes, tant au Québec qu'ailleurs, ont indiqué qu'ils avaient peur d'attraper le sida " (Beazley et al., 1988: 13). Ce taux correspond au $50 \%$ avancé par Sunenblick (Maine, 1988) et au $54 \%$ de Strunin et Hingson (Massachusetts, 1987). Il est supérieur aux pourcentages recueillis dans les régions où l'incidence du sida est faible, comme l'Ohio avec $27 \%$ (Price et al., 1985) et le Texas avec $94 \%$ des jeunes qui ne se considèrent pas comme un groupe à risques (Gottlieb et al., 1988). Mais, il demeure moindre au $64 \%$ identifié à San Francisco (DiClemente et al., 1986). Finalement, " malgré ce qu'ils savent de la maladie et malgré la peur qu'elle engendre, moins de 16 pour cent des élèves de $5^{\mathrm{e}}$ secondaire (11 $\mathrm{e}$ année) ont répondu que la crainte du sida les empêchait d'avoir des relations sexuelles" (Beazley et al., 1988: 13).

\section{La prévention du sida chez les jeunes en regard de leurs activités sexuelles}

Cette mise au point sur les connaissances, les attitudes et les comportements sexuels des jeunes, pointe un défi pour les démarches préventives; quoiqu'ils aient des informations sur le sida (la maladie et ses modes de transmission), les jeunes poursuivent leurs activités sexuelles et, sans condom. Par coriséquent, l'augmentation des connaissances n'encourage pas nécessairement une modification des agirs (IshiiKuntz, 1988). D'ailleurs, une analyse de plus de trente recherches sur le changement de comportements en face du sida chez diverses populations conclut en la nécessité de mieux comprendre le lien entre la connaissance et l'attitude favorisant la réduction des risques (Becker et Joseph, 1988). Mettons de fait en évidence que seulement $50 \%$ des jeunes se sentent directement concernés. Par ailleurs, les renseignements les moins sus, ou retenus, touchent l'incurabilité de la maladie et la longue période 
d'incubation du virus $\mathrm{VIH}$, deux notions qui pourraient accroître la sensation de menace.

Pourtant, des perspectives plus encourageantes apparaissent outre ce sombre portrait de la situation. En effet, des expériences américaines en matière de prévention du sida chez les jeunes identifient des éléments prioritaires pour l'élaboration d'interventions adéquates et efficaces.

Le programme préventif doit tout d'abord présenter un message clair, simple, explicite, approprié à l'âge des interlocuteurs et à leur niveau de connaissances; il doit aussi s'adapter aux références culturelles, au langage, au style de communication et aux modes de vie (DiClemente et al., 1987b). L'objectif est alors d'utiliser un médium qui interpelle les jeunes dans leur réalité quotidienne et qui réduit la distance culturelle avec l'intervenant. Si ce dernier véhicule des émotions, ou plutôt s'il les utilise, cela nécessite un dosage du ton, spécialement s'il est question de peur ; au contraire, l'humour devient un contrepoids adéquat à la diffusion de notions informatives (Baggaley, 1987 ; Edgar et al., 1988).

Autant certains invitent à parler de la sexualité et de ses aspects positifs, de l'amour et du plaisir, pour toucher les jeunes, pour personnaliser la discussion sur le sida et ne pas la limiter à des comportements à modifier (Anatrella, 1989 ; Ciavaldini, 1989), autant d'autres prônent en premier lieu l'abstinence jusqu'au mariage (Centers for Disease Control, 1988). Certes, il importe de noter que «la réduction des risques apparaît plus fréquemment à travers la modification des comportements sexuels et de consommation de drogues qu'à travers leur élimination " (traduction libre, Becker et Joseph, 1988 : 394).

Une campagne sur le sida aurait avantage à introduire un message de promotion de la santé, de lutte plus globale contre les maladies transmissibles sexuellement puisque les gestes préventifs à poser sont similaires (DiClemente et al., 1987b). Cette stratégie vise aussi à déstigmatiser le sida et à le dissocier de la communauté homosexuelle, pour que les hétérosexuels, dont les jeunes, se considèrent également à risques (Edgar et al., 1988). Des leaders peuvent venir légitimer cette nouvelle image sociale et créer une norme en faveur du "safer sex". L'impact d'une telle norme et le support social qui lui est consécutif ont déjà été démontrés comme étant des éléments-clés dans les changements de comportements survenus dans la communauté gaie (Fisher, 1988; Stall, 1988).

On suggère la participation de personnes atteintes qui ont un mode de vie et de pensée ressemblant à celui des jeunes, qui sont proches d'eux, donc significatifs (Dunwoody et al., dans Edgar et al., 1988). Les adolescents peuvent construire eux-mêmes les média de diffusion (Conant et al., 1989) ou devenir des membres actifs des 
programmes préventifs, à l'instar de l'intervention des pairs en matière de contraception (Michaud et Voyer, 1986 ; Tessier, 1986). Que des pairs parlent de prévention du sida s'avère efficace et crédible parce que certaines barrières de générations existant avec des intervenants sont ainsi dépassées (Wendy et Barnes, 1989). Le support du groupe de référence accroît alors l'intégration d'une norme en faveur d'agirs à moindres risques (Melton, 1988) et de l'utilisation du condom (Greenblatt et al., 1989).

Finalement, tout en diffusant des connaissances, ces projets auraient avantage à accentuer le sens des responsabilités et à développer les habiletés afin que ces nouvelles notions soient appliquées (Kaplan, 1988 ; Melton, 1988 ; Greenblath et al., 1989 ; Kipke et Hein, 1989). En ce sens, une attention toute particulière est accordée aux capacités de discuter ouvertement de sexualité, de négocier avec un partenaire, d'affirmer des besoins, de résister à la pression d'autrui et de prendre des décisions rapidement (Baldwin et Baldwin, 1988; Edgar et al., 1988; Flora et Thoreson, 1988 ; Melton, 1988). Certaines mises en situation deviennent ainsi des exercices et des discussions d'alternatives (Conant et al., 1989). Bref, Flora et Thoresen (1988) identifient trois aspects fondamentaux à considérer en prévention du sida chez les jeunes, soit : le cognitif-affectif, mais surtout les habiletés comportementales et le support environnemental.

\section{Le défi de la prévention : un questionnement social plus global}

Cette mise au point par rapport aux jeunes concorde avec d'autres réflexions sur la prévention en matière de sida. Elle s'associe à un questionnement plus global visant une sensibilisation de la population en général, afin que tous se sentent directement concernés et adoptent des agirs sexuels alternatifs; de plus, cette mise au point participe au processus plus précis d'intégration de normes préventives par des groupes-cibles clavantage concernés par la question, de même qu'au processus de changement profond des attitudes chez les personnes présentant des comportements à risques. L'attitude est alors définie en tant que propension structurée et consolidée face à un geste de protection.

Il faut tout d'abord noter l'impact mitigé des campagnes publicitaires. Même si elles influencent les attitudes globales par rapport au sida, elles ont un effet minimal sur les modifications de comportements (Carovano, 1989). Par exemple, l'évaluation de la promotion médiatique faite en Grande-Bretagne révèle de telles conclusions autant auprès de la population (Sherr, 1987) qu'auprès de collégiens ayant reçu un programme 
complémentaire d'informations dans les écoles. On note chez ces étudiants une augmentation des connaissances sur les modes de transmission du VIH, mais sans qu'elle se traduise dans des changements au niveau de leurs agirs sexuels (Clift et Stears, 1988). Ce dernier élément interroge aussi un deuxième moyen utilisé dans la promotion de mesures préventives, soit les programmes visant des groupes-cibles. Le tableau 2 en annexe décrit brièvement cinq recherches évaluatives concernant de tels projets auprès de populations diverses. Quoiqu'un bilan global sur les démarches préventives demeure toujours nécessaire, des généralités peuvent toutefois être dégagées à titre indicatif afin que les expérimentations jusqu'à maintenant tentées deviennent une source d'informations.

Ross et Carson (1988) soulignent la pertinence des programmes adaptés pour atteindre des clientèles spécifiques. Ces programmes soutiennent, de fait, l'acquisition de connaissances et une sensibilisation à des comportements alternatifs. Toutefois, la modification d'attitudes en faveur de l'adoption de ces nouveaux agirs demeure plus complexe et semble notamment dépendre de la clientèle touchée. Par exemple, des homosexuels (Valdiserri et al., 1987) sont davantage ouverts à de tels changements que des femmes vietnamiennes (Flaskerud et al., 1988). Entre autres, cette constatation soulève une fois encore la difficulté qu'a la population hétérosexuelle à s'approprier la problématique du sida, stigmatisée à des groupes à risques, notamment aux homosexuelsbisexuels; à l'instar des jeunes, elle se perçoit en effet peu vulnérable, donc moins concernée (Siegel et Gibson, 1988). Au contraire, la communauté gaie s'est approprié ce problème social qui l'a en premier lieu touchée, puis elle l'a affronté et est intervenue selon ses propres modes d'action.

Malgré les mises en garde sur la portée des démarches préventives jusqu'à présent utilisées, des constatations globales émergent néanmoins de ces quelques expériences et des propos sur les interventions auprès des jeunes. Établir une norme sociale en faveur du geste préventif demeure central pour créer un environnement social supportant le changement individuel. D'autre part, un programme adapté à un groupe-cible doit tenir compte de la culture de ce milieu, des réseaux déjà structurés et des leaders en place; par conséquent, il agit de concert avec cet encadrement afin qu'un support direct soit fourni aux personnes concernées. Ces programmes ne se limitent pas à une diffusion de nouvelles connaissances ; ils insistent sur une acquisition d'habiletés et sur l'investigation des attitudes envers soi-même dans de tels contextes. Ils valorisent ainsi l'accroissement du pouvoir sur soi et du contrôle sur sa réalité ; ces dimensions inhérentes à l'autonomie 


\section{TABLEAU 2}

\section{Un regard sur l'évaluation de programmes de prévention}

\begin{tabular}{|c|c|c|c|c|c|c|c|c|c|c|}
\hline $\begin{array}{c}\text { Devis } \\
\text { et } \\
\text { Recherches }\end{array}$ & $\begin{array}{c}\text { Objet } \\
\text { d'étude }\end{array}$ & $\begin{array}{c}\text { Date } \\
\text { de } \\
\text { parution }\end{array}$ & $\begin{array}{l}\text { Lieu } \\
\text { de } \\
\text { réalisation }\end{array}$ & $\begin{array}{l}\text { Clientèle } \\
\text { visée }\end{array}$ & $\begin{array}{l}\text { Échan- } \\
\text { tillon }\end{array}$ & $\begin{array}{c}\text { Objectif } \\
\text { de } \\
\text { P'intervention }\end{array}$ & $\begin{array}{c}\text { Contenu } \\
\text { du } \\
\text { programme }\end{array}$ & $\begin{array}{c}\text { Cueillette } \\
\text { de } \\
\text { données }\end{array}$ & $\begin{array}{c}\text { Mode } \\
\text { d'analyse }\end{array}$ & Résultats \\
\hline $\begin{array}{l}\text { Valdiserri } \\
\text { et al. }\end{array}$ & $\begin{array}{l}\text { Mesurer la modifi- } \\
\text { cation des atti- } \\
\text { tudes en faveur de } \\
\text { la réduction du } \\
\text { SIDA }\end{array}$ & 1987 & $\begin{array}{l}\text { Pittsburgh, } \\
\text { États-Unis }\end{array}$ & $\begin{array}{c}\text { Homosexuels/ } \\
\text { bisexuels }\end{array}$ & 464 & $\begin{array}{l}\text { Information sur } \\
\text { le SIDA et sur le } \\
\text { condom }\end{array}$ & $\begin{array}{l}1 \text { session de } \\
60-75 \\
\text { minutes par } \\
\text { groupe de } \\
5-10 \\
\text { participants }\end{array}$ & $\begin{array}{l}\text { Devis non } \\
\text { expérimental: } \\
\text { questionnaire } \\
\text { avant-après } \\
\text { retest éventuel }\end{array}$ & $\begin{array}{l}\text { - Quantitatif à } \\
\text { partir des corré- } \\
\text { lations, à l'aide } \\
\text { d'une échelle } \\
\text { d'attitude }\end{array}$ & $\begin{array}{l}\text { - Bonnes connaissances sur le } \\
\text { SIDA au départ mais pour } 60 \% \\
\text { poursuite des comportements à } \\
\text { risques } \\
\text { - Meilleures attitudes à l'égard du } \\
\text { SAFER SEX après la rencontre }\end{array}$ \\
\hline $\begin{array}{l}\text { Magura } \\
\text { et al. }\end{array}$ & $\begin{array}{l}\text { Identifier l'impact } \\
\text { d'un groupe de } \\
\text { prévention }\end{array}$ & 1988 & $\begin{array}{l}\text { New York } \\
\text { États-Unis }\end{array}$ & Toxicomanes & 69 & $\begin{array}{l}\text { Information sur } \\
\text { le SIDA et sur le } \\
\text { danger des } \\
\text { échanges de } \\
\text { seringues }\end{array}$ & $\begin{array}{l}4 \text { rencontres } \\
\text { de } 2 \text { heures } \\
\text { par groupe } \\
\text { de } 10 \text { parti- } \\
\text { cipants }\end{array}$ & $\begin{array}{l}\text { - Devis non } \\
\text { expérimental } \\
\text { avant-après } \\
\text { - Discussion } \\
\text { d'évaluation }\end{array}$ & $\begin{array}{l}\text { - Quantitatif avec } \\
\text { questionnaire } \\
\text { d'évaluation } \\
\text { - Qualitatif à l'aide } \\
\text { des propos } \\
\text { échangés }\end{array}$ & $\begin{array}{l}\text { - Dans le cadre d'un programme } \\
\text { de methadone pour arrêter la } \\
\text { drogue } \\
\text { - Mise en valeur du support mu- } \\
\text { tuel }\end{array}$ \\
\hline $\begin{array}{l}\text { Chikwem } \\
\text { et al. }\end{array}$ & $\begin{array}{l}\text { Évaluer les } \\
\text { attitudes à } \\
\text { l'égard du SIDA }\end{array}$ & 1988 & Nigéria & $\begin{array}{c}\text { Femmes } \\
\text { (prostituées) }\end{array}$ & 767 & $\begin{array}{l}\text { Information sur } \\
\text { le SIDA et sensi- } \\
\text { bilisation à leur } \\
\text { rôle pour une } \\
\text { prévention }\end{array}$ & $\begin{array}{l}\text { Une série de } \\
\text { rencontres } \\
\text { par sous- } \\
\text { groupes }\end{array}$ & $\begin{array}{l}\text { Devis non } \\
\text { expérimental: } \\
\text { questionnaire } \\
\text { après seulement }\end{array}$ & $\begin{array}{l}\text { - Quantitatif avec } \\
\text { questionnaire } \\
\text { sur les compor- } \\
\text { tements éven- } \\
\text { tuels }\end{array}$ & $\begin{array}{l}\text { - Informations sur leurs activités } \\
\text { de prostitution } \\
\text { - Ouverture à utiliser le condom } \\
\text { après la session de sensibilisa- } \\
\text { tion }\end{array}$ \\
\hline
\end{tabular}




\begin{tabular}{|l|l|l|l|l|l|l|l|l|l|l|}
$\begin{array}{l}\text { Flaskerud } \\
\text { et al. }\end{array}$ & $\begin{array}{l}\text { Mesurer l'acquisi- } \\
\text { tion de connais- } \\
\text { sances et la modi- } \\
\text { fication d'attitudes }\end{array}$ & 1988 & $\begin{array}{l}\text { Los Angeles } \\
\text { Etats-Unis }\end{array}$ & $\begin{array}{l}\text { Femmes } \\
\text { (partenaires) }\end{array}$ & 369 & $\begin{array}{l}\text { Information sur } \\
\text { le SIDA, le } \\
\text { SAFER SEX et la } \\
\text { protection en } \\
\text { cas de parte- } \\
\text { naires IVDU } \\
\text { (consommateurs } \\
\text { de drogues } \\
\text { intraveineuses) }\end{array}$ & $\begin{array}{l}1 \text { rencontre } \\
\text { de 60-75 } \\
\text { minutes } \\
\text { par groupe } \\
\text { de 50 } \\
\text { participants }\end{array}$ & $\begin{array}{l}\text { Devis } \\
\text { expérimental: } \\
\text { avant-après } \\
\text { groupe-contrôle }\end{array}$ & $\begin{array}{l}\text { - Quantitatif avec } \\
\text { échelle de } \\
\text { connaissances et } \\
\text { d'attitudes }\end{array}$ & $\begin{array}{l}\text { - Recherche auprès de réfugiées } \\
\text { vietnamiennes bénéficiaires } \\
\text { d'aide sociale } \\
- \text { Acquisition de connaissances } \\
\text { mais peu de changements d'at- } \\
\text { titudes }\end{array}$ \\
\hline $\begin{array}{l}\text { Ross et } \\
\text { Carson }\end{array}$ & $\begin{array}{l}\text { Mesurer les atti- } \\
\text { tudes par rapport } \\
\text { au SIDA en consi- } \\
\text { dérant la source } \\
\text { d'information }\end{array}$ & 1988 & Australie & Population & 2601 & $\begin{array}{l}\text { Information sur } \\
\text { le SIDA et les } \\
\text { mesures } \\
\text { préventives }\end{array}$ & $\begin{array}{l}\text { - Publicité } \\
\text { médiatique } \\
\text { - Brochures } \\
- \text { Contacts } \\
\text { directs }\end{array}$ & $\begin{array}{l}\text { Devis non expé- } \\
\text { rimental: après } \\
\text { seulement } \\
\text { auprès d'un } \\
\text { échantillon } \\
\text { représentatif }\end{array}$ & $\begin{array}{l}\text { - Quantitatif avec } \\
\text { questionnaire } \\
\text { sur les compor- } \\
\text { tements à ris- } \\
\text { ques }\end{array}$ & $\begin{array}{l}\text { - Les personnes à risques n'écou- } \\
\text { tent pas davantage les messages } \\
\text { médiatiques, mais elles sont } \\
\text { ouvertes lors de contacts directs } \\
\text { avec des intervenants }\end{array}$ \\
\hline
\end{tabular}


sont fondamentales dans un processus de prise en charge d'un changement dans l'agir sexuel, comportant à la fois une forte intensité émotive et une négociation avec autrui, à travers des enjeux de pouvoir.

En guise de conclusion, il importe de mettre en lumière qu'à travers cette discussion, la prévention interpelle la "révolution sexuelle des années 70 ». Certains voient d'un œil favorable cette remise en question des comportements sexuels pour réintroduire des valeurs traditionnelles. Par contre, elle ouvre aussi la porte à l'exploration d'alternatives, tant au niveau de la sexualité que sur le plan des modes de vie.

\section{Références bibliographiques}

AnAtrella, Tony (1989), "Le Sida, les jeunes et l'état des sexualités " dans Psychologie du SIDA, Bruxelles: Pierre Mardaga, p. 219-233.

BAGGALEY, J.P. (1988), " Perceived effectiveness of international AIDS Campaigns ", Health education research, vol. 3, no 1, p. 7-17.

BALDWIN, John et Janice BALDWIN (1988), "Factors affecting AIDS-related sexual risk-taking behavior among college students ", The Journal of sex research, vol. 25, no 2, p. 181-196.

BAuM, Andrew et Sarah Nesselhof (1988), "Psychological research and the prevention, etiology and treatment of AIDS ", American Psychologist, vol. 43, no 11, p. 900-906.

BEAZLEY, Richard et al. (1988), L'étude sur les jeunes Canadiens face au SIDA, résultats pour le Québec, Kingston : Université Queen's.

BECKER, Marshall et Jill JOSEPH (1988), "Aids and behavioral change to reduce risk : a review ", American journal of public health, vol. 78, no 4, p. 394-410.

Bellefeuille, Roger (1989), " Les jeunes Québécois et le SIDA : similitudes et écarts avec ceux du reste du Canada ", Le Soleil, mercredi le 15 février 1989, p. A7.

CAROVANO, Kathryn (1989), Education and evaluation : partners in AIDS prevention, Washington: Academy for educational development.

CAUCHON, Paul (1989), "Le SIDA n'est plus “la maladie des autres", l'épidémie se répand sur tous les continents et sévit dans tous les milieux", Le Devoir, samedi le 27 mai, p. A7.

Centers for Disease Control (1988), "Guidelines for effective school health education to prevent the spread of AIDS", New York State journal of medicine, vol. 88, no 5, p. 266-272.

CHIKWEM, John (1988), "I Impact of health education on prostitutes awareness and attitudes to acquired immune deficiency syndrome (AIDS), Public Health, vol. 100, no 5, p. 439-445.

CIAVALDINI, A. (1989), " "L'impossible condom" ou les avatars du préservatif » dans Psychologie du SIDA, Bruxelles: Pierre Mardaga, p. 251-265.

CuIf Stephen et David SteARS (1988), "Beliefs and attitudes regarding AIDS among British college students : a preliminary study of change between 
November 1986 and May 1987 ", Health education research, vol. 3, no 1, p. 75-88.

CONANT, Sloane, Beverlie (1989), "AIDS education in Darmouth", remis à la Ve conférence sur le SIDA à Montréal, Hanover: Darmouth college (texte inédit).

DiClemente, Ralph, Jim ZoRn et Lydia TeMOSHOK (1986), « Adolescents and AIDS : a survey of knowledge attitudes and beliefs about AIDS in San Francisco ", American journal of public health, vol. 76, no 12, p. 1443-1445.

DiClemente, Ralph, Jim ZORN et Lydia TeMOSHOK (1987), "The association of gender, ethnicity, and length of residence in the Bay area to adolescents knowledge and attitudes about acquired immune deficiency syndrome", Journal of applied social psychology, vol. 17, p. 216-230.

DiClemente, Ralph, Cherrie B. Boyer et Stephen Mills (1987), "Prevention of aids among adolescents : strategies for the development of comprehensive riskreduction health education programs ", Health education research, vol. 2, n० 3, p. 287-291.

Desrosiers, Marie (1986), "La sexualité dans le contexte normal de l'adolescence des années 80 ", Intervention, no 73, p. 24-30.

Drolet, Marie (1989), "Un aperçu des aspects psychosociaux du SIDA et un regard sur les recherches évaluatives", travail présenté dans le cadre du Séminaire de doctorat II, Québec : École de Service Social, Université Laval (texte inédit).

DUPRAS, André et al. (1989), La sexualité des Montréalais et le SIDA, Longueuil : Éditions IRIS.

Edgar, Timothy, Vicki Freemuth et Sharon Hammond (1988), "Communicating the AIDS risk to college students: the problem of motivating change", Health education research, vol. 3, no 1, p. 59-65.

FILION Françoise (1986), Influence du foyer de contrôle sur l'utilisation de la contraception par des adolescents(es), mémoire de maîtrise présenté à la Faculté des sciences infirmières, Université de Montréal (texte inédit).

FISHER, Jeffrey (1988), "Possible effects of reference group and social influence on AIDS-risk behavior and AIDS prevention ", American psychologist, vol. 43, no 11, p. 914-920.

FLASKERUD, Jacquelyn et al. (1988), "An AIDS education program for Vietnamese women ", New York State journal of medicine, vol. 88, n० 12, p. 632-637.

FLORA, June et Carl THORESEN (1988), "Reducing the risk of aids in adolescents", American psychologist, vol. 43, no 11, p. 965-970.

FrAPPIER, Jean-Yves (1980), "Adolescence et sexualité ", conférence donnée en 1985 au colloque sur Sexualité et adolescence organisé par le Département de Santé Communautaire du Centre Hospitalier Sainte-Marie de TroisRivières (texte inédit).

Goodwin, Megan et Bruce Roscoe (1988), "AIDS: students' knowledge and attitudes at a Midwestern university ", Journal of American College health, vol. $36, n^{\circ} 4$, p. 214-222.

GotTLieB, Nell et al. (1988), "AIDS-related knowledge, attitudes, behaviors and intentions among Texas College students", Health education research, vol. $3, n^{\circ} 1$, p. 67-73. 
Greator, lan et John PACKer (1989), "Sexual behavior in University Students : report of a postal survey ", Public Health, vol. 103, p. 199-203.

GreenBLATt, Ruth et al. (1989), "Predictors of condom use and AIDS in a group of sexually active adolescent women », résumé de présentation à la Ve conférence sur le SIDA, Montréal.

HeIN, Karen (1987), "Aids in adolescents : a rational for concern ", New York State journal of medicine, vol. 87, n० 5, p. 290-295.

ISHII-KUNTZ, Masako (1988), "Acquired immune deficiency syndrome and sexual behavior changes in a college student sample ", Sociology and social research, vol. 73, no 1, p. 13-18.

KING, A.J.C. et al. (1988), Étude sur les jeunes Canadiens face au Sida, Kingston : Université Queen's.

KIPKE, Michele, C. BOYER et K. HeIN (1989), "An evaluation of an AIDS risk reduction education and skills training (ATSREST) program for adolescents ", résumé de présentation à la Ve conférence sur le SIDA, Montréal.

LÉVESQUE, Lia (1989), "Aux États-Unis, l'infection se répand chez les adolescents ", Le Devoir, mardi 6 juin, p. 7.

MAGURA, Stephen et al. (1988), "Education/support groups for AIDS prevention with at-risk clients ", Social Casework, vol. 70, n० 1, p. 10-20.

MeLton, Gary (1988), "Adolescents and prevention of AIDS ", Professional psychology research and practice, vol. $19, n^{\circ} 4$, p. 403-408.

Michaud, Marie-Claude et Johanne Vorer (1986), "Multi-jeunesse : une expérimentation d'entraide par les pairs", dans Jeunesse et Sexualité : actes du colloque, Longueuil : Éditions IRIS, p. 547-555.

Price, J.M., S. DeSmOND et G. KUKALKA (1985), " High school students' perceptions and misperceptions of AIDS ", Journal of school health, vol. 55, p. 107-109.

Ross, Michael (1988), "Personality factors that differenciate homosexual men with positive and negative attitudes toward condom use ", New York State journal of medicine, vol. 88, n० 12, p. 626-628.

Ross, Michael et James CARSON (1988), «Effectiveness of distribution of information on AIDS ; a national study of six media in Australia ", New York State journal of medicine, vol. 88, no 5, p. 238-241.

SHAYNE, Visian et Barbara KAPLAN (1988), "Aids education for adolescents », Youth and Society, vol. 20, no 2, p. 180-208.

SHERR, L. (1987), " An Evaluation of the UK Government Health Education campaign on Aids", Psychology and Health, vol. 1, p. 61-72.

Siegel, Karolynn et William Gibson (1988), "Basics to the modification of sexual behavior among heterosexuals at-risk for acquired immunodeficiency syndrome ", New York State journal of medicine, vol. 88, no 2, p. 66-70.

STALL, Ron D. et al. (1988), "Behavioral risk reduction for HIV infection among boy and bisexual men ", American psychologist, vol. 43, no 11, p. 878-885.

STRUNIN, L. (1989), "Do Health belief model beliefs about HIV predict adolescent condom use?", résumé de présentation à la Ve conférence sur le SIDA, Montréal. 
STRUNIN, Lee et Ralph HINGSON (1987), "Acquired immunodeficiency syndrome and adolescents : knowledge, beliefs attitudes and behaviors ", Pediatrics, vol. 79, no 5, p. 825-828.

SUNENBLICK, Mary Beth (1988), "The AIDS epidemic : sexual behaviors of adolescents ", South college studies in Social work, vol. 59, no 1, p. 21-38.

TESSIER, Monique (1986), "Sexualité adolescente et prévention : des choix s'imposent ", dans Jeunesse et Sexualité ; actes du colloque, Longueuil : Éditions IRIS, p. 528-547.

VALDISERRI, Ronald et al. (1987), "The effect of group education on improving attitudes about AIDS risk reduction", dans New York State journal of medecine, vol. 87, n० 5, p. 272-277.

Wendy, Arnold et F. BARNeS (1989), "Peer education program reaches High risk adolescents with AIDS information and prevention ", résumé de présentation à la Ve conférence sur le SIDA, Montréal.

WILKINS, Jean (1985), "La sexualité durant l'adolescence; phénomène pluridimensionnel " dans Médecine de l'adolescence : une médecine spécifique, Montréal : Hôpital Sainte-Justine, p. 125-182. 